\title{
Role of normal sleep and sleep apnea in human memory processing
}

\author{
Shilpi Ahuja' \\ Rebecca K Chen' \\ Korey Kam' \\ Ward D Pettibone' \\ Ricardo S Osorio ${ }^{2}$ \\ Andrew W Varga'
}

'Mount Sinai Integrative Sleep Center, Division of Pulmonary, Critical Care, and Sleep Medicine, Icahn School of Medicine at Mount Sinai, New York, NY, USA; ${ }^{2}$ Center for Brain Health, Department of Psychiatry, NYU School of Medicine, New York, NY, USA
This article was published in the following Dove Press journal: Nature and Science of Sleep

\begin{abstract}
A fundamental problem in the field of obstructive sleep apnea (OSA) and memory is that it has historically minimized the basic neurobiology of sleep's role in memory. Memory formation has been classically divided into phases of encoding, processing/consolidation, and retrieval. An abundance of evidence suggests that sleep plays a critical role specifically in the processing/consolidation phase, but may do so differentially for memories that were encoded using particular brain circuits. In this review, we discuss some of the more established evidence for sleep's function in the processing of declarative, spatial navigational, emotional, and motor/procedural memories and more emerging evidence highlighting sleep's importance in higher order functions such as probabilistic learning, transitive inference, and category/gist learning. Furthermore, we discuss sleep's capacity for memory augmentation through targeted/ cued memory reactivation. OSA - by virtue of its associated sleep fragmentation, intermittent hypoxia, and potential brain structural effects - is well positioned to specifically impact the processing/consolidation phase, but testing this possibility requires experimental paradigms in which memory encoding and retrieval are separated by a period of sleep with and without the presence of OSA. We argue that such paradigms should focus on the specific types of memory tasks for which sleep has been shown to have a significant effect. We discuss the small number of studies in which this has been done, in which OSA nearly uniformly negatively impacts offline memory processing. When periods of offline processing are minimal or absent and do not contain sleep, as is the case in the broad literature on OSA and memory, the effects of OSA on memory are far less consistent.
\end{abstract}

Keywords: consolidation, learning, REM sleep, sleep spindles, slow oscillations

\section{Introduction}

Over the last few years, there have been many publications exploring the reemerging interest in the multifaceted relationship between sleep and memory. This, however, is not a new concept. In 1900, Muller and Pilzecker first theorized that new information required a process of consolidation before it could assume a permanent form ${ }^{1}-\mathrm{a}$ process that was later recognized to be enhanced after a night's sleep. ${ }^{2}$

Memory is comprised of three interdependent processes: encoding, processing/ consolidation, and retrieval. Encoding allows for the formation of a memory trace in response to a new stimulus, which then undergoes a process of stabilization, strengthening, and integration into existing knowledge networks (consolidation). This information is then accessible for easy recall (retrieval). ${ }^{3}$ Memory is traditionally divided into two different categories - declarative and non-declarative memory - and each of them depends on specific neural interactions to mediate their processes. Declarative
Correspondence: Andrew W Varga Mount Sinai Integrative Sleep Center, Division of Pulmonary, Critical Care, and Sleep Medicine, Icahn School of Medicine at Mount Sinai, One Gustave L. Levy Place, Annenberg 2I-44, New York, NY 10029, USA

Tel +l 2I2 24I 6084

$\mathrm{Fax}+\mathrm{I} 2125230498$

Email andrew.varga@mssm.edu 
memory is explicit, associated with awareness, and can be subdivided into memories associated with specific context that includes some element of time (episodic memory) and fact-based information (semantic memory). ${ }^{4}$ Non-declarative memory, on the other hand, is often linked with information or abilities not associated with conscious awareness.

Sleep, too, is not a homogeneous entity and encompasses different neurochemical states and neural processes characterizing varied sleep stages. It is well described in the literature that sleep facilitates memory consolidation and confers a memory-retention advantage compared to equivalent periods of time awake for many, but not necessarily all, types of memory. It is not out the realm of possibility, then, to expect that when sleep is disrupted, the natural processes of memory consolidation may, therefore, be altered or impaired. In the current age, with its high prevalence of sleep disorders - including widespread sleep deprivation and poor sleep hygiene - identifying the impact of these ailments on memory and their treatment is vital. ${ }^{5,6}$ Obstructive sleep apnea (OSA) - a chronic condition in which repetitive upper airway collapse results in intermittent hypoxia and sleep fragmentation - provides the perfect lens through which to explore the interaction of a clinical sleep disorder with memory. Moreover, OSA is exceedingly common. The OSA syndrome (OSAS) occurs when there are a minimum number of apneas and hypopneas per hour associated with daytime symptoms and it has an estimated prevalence of $4 \% .^{7}$ However, when OSA is defined solely by having an apnea-hypopnea index (AHI3a) $>15 /$ hour - where hypopneas are defined as terminating in either a $\geq 3 \%$ drop in oxygen saturation or arousal - the prevalence can be as high as $50 \%$ in certain populations, especially older individuals. ${ }^{8,9}$

In this review, we aim to provide an overview of the current literature demonstrating the unique influence of individual sleep stages, specific electroencephalogram (EEG) oscillatory frequencies, and their bidirectional modulation on different types of memory as well as to highlight the dynamic and complex interplay between memory and sleep, principally in human subjects. We then review how interruption of these complex processes in OSA sheds light upon the relationship between the two.

\section{Declarative/episodic memory}

The mechanistic understanding of sleep's role in memory processing was greatly influenced by a series of seminal papers describing hippocampal encoding of space and the reemergence of patterns of firing of hippocampal cell ensembles during sleep that had occurred during periods of prior active wake states. ${ }^{10,11}$ Consistent with this hippocampal model, neuronal reexpression/replay in neocortical regions during sleep has also been observed to mimic preceding awake experiences. ${ }^{12,13}$ These observations have helped to establish a model in which reactivation of newly encoded information during sleep is thought to mediate the redistribution and transfer from short-term storage within the hippocampus to the long-term stores of the neocortex, where it can then be integrated into preexisting knowledge and easily retrieved. ${ }^{14,15}$ This process may be broken into two stages. In the "exploration" stage in the awake state, new information enters CA3 pyramidal cells of the hippocampus from the entorhinal cortex for temporary storage. During sleep, this information is then "replayed" - primarily during hippocampal sharp wave ripples during slow-wave sleep (SWS). It has been postulated that the temporal coordination of neocortical sleep spindles with hippocampal sharp wave ripple complexes during SWS could facilitate the transfer of information between the hippocampus and neocortex that could help support memory. ${ }^{12,16-19}$ Although replay has not been observed at the cellular level in human subjects, certain high-frequency cortical EEG motifs have been observed to occur in non-rapid eye movement (NREM) sleep in humans, ${ }^{20}$ and blood oxygen level dependent (BOLD) signals recorded with functional magnetic resonance imaging (fMRI) during both encoding and subsequent sleep relate to declarative memory in humans. ${ }^{21}$

Simple declarative memory testing in human subjects is often performed using word-pair or face/object recall tasks. Numerous studies have shown improved recall of newly formed memories following sleep. ${ }^{22,23}$ In the case of declarative memory, NREM sleep - and, specifically, SWS - is thought to be the predominant stage in which memory consolidation occurs. Despite comparable initial learning, it has been shown that subsequent sleep improves recall of paired associate word lists, with a distinct difference noted after early SWS-predominant - compared to late REM-predominant - sleep. ${ }^{23}$ This finding has been reiterated in other studies, confirming the importance of SWS (independent of time of day) in the consolidation of declarative memory, ${ }^{14,24-26}$ including a study suggesting that frontal slow-wave activity statistically mediates the association between prefrontal cortical volume and declarative word-pair memory with aging. ${ }^{27}$ Although these studies largely correlate memory with the occurrence of sleep, targeted disruption of SWS with auditory tones has also been shown to impair declarative memory consolidation ${ }^{28}$ and, conversely, augmentation of slow-wave activity with transcranial stimulation has been shown to improve declarative memory performance in both younger ${ }^{29,30}$ 
and older ${ }^{31}$ subjects. Phase-locking auditory stimuli to the up states of slow waves can increase slow-wave and spindle activity and improve word-pair memory. ${ }^{32}$ Augmentation of slow-wave activity with sodium oxybate, ${ }^{33}$ baclofen, ${ }^{33}$ or tiagabine ${ }^{34}$ failed to improve declarative memory performance across sleep versus placebo, suggesting that slow-wave timing may be critical or that these drugs have pleiotropic effects, some of which counter the presumed benefit of slowwave augmentation.

The importance of the temporal relationship between sleep onset and learning remains controversial. It has been suggested that sleep must occur immediately after learning to benefit performance and prevent degradation in memory trace. However, other studies suggest that recall performance following delayed sleep onset after learning is still superior to that in awake states, promoting the idea that the sleep has an active role in memory processing. ${ }^{35}$

It has been debated whether the beneficial effects of sleep on memory as described are explained by reduced memory deterioration/protection from interference with sleep or actively contribute to improvement in memory itself. ${ }^{22}$ The interference theory suggests that both sleep and quiet wake states would augment memory in comparison to an equivalent period of active wake. When declarative memory across these conditions was formally tested, subjects in an "active wake" group who sat on a busy street and discussed various topics with the experimenter had equivalent declarative memory performance as that of a "reduced interference" group who meditated in a quiet room before memory retesting. Both of these conditions were inferior to a period of sleep, ${ }^{36}$ supporting the idea that sleep's role is more than simple protection from interference.

It has been shown that sleep plays a vital role not only in post-memory processing, but also provides the human brain with essential preparation for next-day memory formation. Pre-training sleep deprivation causes a marked disruption in hippocampal activity, resulting in impaired learning acquisition. ${ }^{37-39}$ Further, according to the synaptic homeostasis hypothesis, accrued time awake and ongoing encoding of new information decreases learning capacity that can be restored only through sleep. ${ }^{40,41}$ Whereas circadian timing could influence encoding ability, the effect of circadian timing appears to be influenced by prior sleep. Episodic face-name encoding was worse at 18:00 compared to 12:00 when no sleep occurred prior to training. However, encoding was actually better at 18:00 than 12:00 when an equivalent period of sleep occurred prior to these training sessions, and episodic encoding capacity correlated with frontal fast spindles during the period of preceding sleep. ${ }^{40}$
This role for sleep spindles is, perhaps, not surprising given the theories suggesting that it is the coordinated activity of spindles and slow waves that support memory. Moreover, spindles have been shown to boost neocortical synaptic responses, which may provide a mechanism for enhanced memory consolidation. ${ }^{42,43}$ This study, along with others, suggests that the analysis of electrophysiologic features during sleep may provide further insight on the role of Stage 2 sleep in the role of declarative memory as well. Significantly increased sleep spindle density was observed following a 1-hour declarative task as compared to a non-learning task and, here too, improved recall performance was positively associated with spindle density. ${ }^{43}$

\section{Spatial learning/memory}

Spatial memory can be defined in various ways, including the ability to remember the spatial location of object pairs organized in a $2 \times 2$ grid akin to children's games ${ }^{44}$ or the ability to recall complex three-dimensional (3D) objects displayed in novel orientations after encoding. ${ }^{45}$ However, because of the clear parallels to the spatial memory tasks that rodents are capable of performing, the most common form of spatial memory assessment in human subjects is spatial navigational memory - defined as the ability to recall routes to salient targets in context-specific environments. Spatial navigational memory is responsible for maintaining information with regard to a person's environment and orientation and has clear clinical relevance to Alzheimer's dementia - where deficits in spatial orientation can be one of the earliest cognitive hallmarks of the disease. ${ }^{46}$

Though its neural underpinnings are clearly complex, involving multiple brain areas, spatial navigational memory is thought to be subserved by at least two brain systems working in concert: an allocentric system mediated largely by medial temporal lobe structures including the hippocampus and entorhinal cortex, wherein external landmarks are used to create a mental spatial map, and an egocentric system mediated largely by the striatum, composed of the caudate and putamen, which incorporates body movement, turn direction, speed, and elapsed time. ${ }^{47-49}$ Across sleep, it is thought that information encoded by the hippocampus gets integrated and distributed to cortical structures, such that there is less activation of the hippocampus and possibly more activation of the striatum ${ }^{50}$ upon subsequent experience in the same environment - a process known as systems consolidation.

A role for sleep in spatial navigational memory is supported by studies demonstrating enhanced navigational performance on a $3 \mathrm{D}$ virtual navigation task after a night 
of overnight sleep in comparison to an equivalent period of natural daytime wakefulness, ${ }^{51}$ or in comparison to fully maintaining wakefulness overnight (i.e., sleep deprivation). ${ }^{52}$ Related benefits on spatial navigational performance have also been observed across a daytime nap, and this benefit appears to be positively influenced by dream recall of the maze environment during the nap ${ }^{53}$ as well as positively influenced by prior exposure to $3 \mathrm{D}$ first-person-experience video games. $^{54}$

A specific role for slow waves in this process is supported by observations suggesting that navigational performance improvement across a nap significantly correlates with relative slow-wave activity during N2 sleep during a nap ${ }^{54}$ and also with relative frontal slow-wave activity during NREM sleep across overnight sleep. ${ }^{55}$ This observation could account for a reduced benefit of overnight sleep on spatial navigational memory processing in older subjects as SWS declines with age. Additionally, brain fMRI studies during a period of sleep following spatial navigational encoding demonstrated that, in a limited number of subjects, the degree of blood flow to the right hippocampus during SWS correlated with subsequent improvement in spatial navigation performance. ${ }^{56}$

A mechanistic understanding of a role for REM sleep in spatial navigational memory is less complete than the current understanding of the role for NREM sleep; however, several studies in rodents indicate REM sleep suppression impaired spatial memory in the Morris water maze $\mathrm{e}^{57-62}$ and other spatial tasks. ${ }^{63,64}$ Furthermore, optogenetic disruption of hippocampal theta power during REM sleep - but not NREM sleep - impaired spatial memory in mice. ${ }^{65}$ In human subjects, the possible role for REM sleep is underexplored. Although REM sleep constituted only a tiny fraction of sleep during the nap study, the observation that dreaming of the spatial environment during the nap significantly augmented post-sleep navigation performance ${ }^{53}$ raised the possibility that REM during overnight sleep - when most of active dreaming occurs - might have a role in spatial navigational memory processing. Such a possibility was explicitly tested by inducing OSA exclusively during REM sleep through CPAP withdrawal in subjects with known severe OSA who used CPAP regularly. ${ }^{66}$ The benefits of sleep on spatial navigational performance were significantly attenuated by REM OSA, and overnight performance change correlated with measures of REM fragmentation (but not REM amount). The observations that the highest activity in the striatum is observed during REM sleep - as measured by blood flow in human subjects ${ }^{67}$ or by neuronal unit activity ${ }^{68}$ or c-fos immediate early gene expression $^{69}$ in animal models - and the observations that acute sleep deprivation impairs appropriate recruitment of the striatum for navigational performance ${ }^{50,70}$ suggests that REM sleep may be important for this plasticity to occur.

\section{Emotional memory}

Emotionally arousing stimuli consistently and significantly enhance memory acquisition compared to neutral stimuli - an effect that is facilitated by adequate sleep. ${ }^{71-74}$ Furthermore, emotional memories have a certain distinctness and persistence that other types of memory seem to lack. It has been postulated that beta-adrenergic stimulation at the initial moment of learning plays a key role in mediating this effect. ${ }^{74}$ In support of this, studies in which adrenergic blockers, such as propranolol, are administered shows interference with the emotional enhancement. ${ }^{75,76}$ Adrenergic stimulation likely acts through activation of the amygdala. ${ }^{73}$ Activation in the amygdala and the simultaneous response described in the visual cortex in response to emotional stimuli may provide a mechanism by which enhanced perceptual coding is seen in emotional events. ${ }^{77,78}$ The amygdala - through its communication with the hypothalamus - then facilitates memory consolidation. $^{78}$

The role of sleep in the processing of emotional memory is adaptive and complex. Studies have been conducted to investigate how different components of an emotional memory can change over periods of sleep versus wake states. A study in which subjects were presented with scenes depicting objects against neutral backgrounds and then re-tested after a period of sleep revealed selective preservation of negative objects but not their accompanying backgrounds. ${ }^{79}$ Such a phenomenon did not occur across a period of natural daytime wake, ${ }^{80}$ but did occur across a 90 -minute daytime nap. ${ }^{81}$ Furthermore, brain fMRI studies revealed that the left amygdala and right parahippocampus responded more strongly during correct memory for emotional contexts post-overnight sleep. This highlights the concept of selective preservation of emotional memories: the idea that sleep "unbinds" representation of experience and preferentially preserves the most salient or emotional aspects at the expense of what may be considered less important features. ${ }^{72,82,83}$ This finding is supported by other studies, ${ }^{82}$ and it is felt that whereas consolidation starts in the initial period of sleep, it intensifies over time with protection of the hallmark features of emotional memory, but with further deterioration of other associated background information.

In contrast to pure declarative memory, a weight of evidence supports that REM sleep modulates emotional memory sleep processing. ${ }^{84}$ Enhanced retention of emotional text was 
associated with late-night sleep - a period that is well known to be rich in REM sleep. ${ }^{84,85}$ Moreover, it has been shown that post-sleep emotional memory recall is significantly associated with the amount of REM sleep obtained, but not with total sleep time or time in any other sleep stage. ${ }^{71}$ Furthermore, the amount of EEG theta activity that is highly expressed in REM specifically predicts post-sleep emotional memory recall. ${ }^{71}$ Brain fMRI studies indicated that overnight sleep results in increased connectivity between the amygdala and ventromedial prefrontal cortex following exposure to an emotional stimulus. ${ }^{86}$ This increased connectivity was paralleled by an overall decrease in amygdala reactivity following sleep, and decreased gamma power during REM sleep was significantly associated with a decrease in subjective emotional reactivity ratings. ${ }^{87}$

\section{Motor/procedural learning}

Motor/procedural learning is classically thought to be a form of non-declarative learning, such as learning to ride a bicycle or play a musical instrument, in human subjects. One of the implications of this classification is that this type of learning typically bypasses the hippocampus; however, recruitment of the hippocampus for motor tasks does sometimes occur, ${ }^{88,89}$ and depends on the precise motor task. Interestingly, it has been argued that the specific involvement of the hippocampus in motor tasks is a prerequisite for sleep-dependent consolidation of motor learning to occur; ${ }^{90}$ however, this remains controversial.

A role for sleep in the processing of motor learning has been observed for several motor tasks, including continuous tracking/pursuit rotor tasks ${ }^{91,92}$ and forms of motor adaptation such as mirror-tracing ${ }^{23,93}$ or joystick/computer mouse adaptation tasks, ${ }^{94,95}$ where subjects are required to compensate for externally applied actual or perceived forces requiring movement trajectory changes in order to successfully complete the initially intended reaching movement. However, by far the most common motor learning task in which a role for sleep has been assessed is a finger-tapping motor sequence task (MST). The most common form of this task involves tapping a novel five-element sequence with the nondominant hand repetitively as quickly as possible in 30-second bins with 30 seconds of rest. This pattern is typically repeated 12 times at any one time point. It should be noted that this version of the task is learned explicitly, as the exact sequence is known to the subject throughout. A related version of this task is called the serial reaction time test, where the sequence is visually cued on a screen, and the pattern may or may not be accessible to awareness. When subjects are unaware of the underlying pattern, a form of implicit learning can occur.

Numerous studies have demonstrated a benefit of sleep on subsequent performance compared to an equivalent time awake when the standard explicit MST is employed ${ }^{96,97}$ (see King et al, ${ }^{90}$ for a thorough review). The effect of sleep may be moderated by age, with weaker effects observed in both children $^{44}$ and elderly subjects. ${ }^{98}$ The effect of sleep appears to be greater with increasing motor-task complexity ${ }^{99}-$ an observation recapitulated in animal models. ${ }^{100,101}$ Interestingly, dissociable "spatial goal" and "motor sequence" components to MSTs have been identified, with evidence suggesting that the spatial component is enhanced by sleep whereas the sequential component is enhanced by daytime wake states. ${ }^{102}$

Evidence suggests that there are potential roles for both REM and NREM sleep in procedural motor memory processing. Much of the early work on sleep and motor learning focused on REM sleep where post-training increases in REM sleep or its associated features after learning a complex motor task ${ }^{103,104}$ implicated REM-related mechanisms in offline procedural memory consolidation. Additionally, positron emission tomography imaging during REM sleep demonstrated both reactivation ${ }^{105}$ of - and increased connectivity $^{106}$ between - motor-task-relevant areas. Furthermore, increasing premotor cortical excitability during REM with transcranial direct current stimulation (tDCS) improved postsleep motor-task performance. ${ }^{107}$ Conversely, studies show impaired memory recall for a procedural task following REM sleep deprivation and total sleep deprivation, although REM deprivation in the first vs. second half of the night shows no difference in the degree of impairment, suggesting that the benefits of REM are not restricted to a specific "window" of time. ${ }^{103,108}$ Use of an anticholinergic agent impairs REM sleep and procedural memory consolidation, supporting the hypothesis that REM-sleep-dependent processing may be facilitated specifically by the high cholinergic state characteristic of REM sleep. ${ }^{109}$

Thus, it came as an initial surprise to investigators that utilizing selective serotonin reuptake inhibitors (SSRIs) or serotonin-norepinephrine reuptake inhibitors (SNRIs) - known REM suppressants - did not impair procedural memory consolidation. ${ }^{110,111}$ However, one consequence of REM suppression was an increase in NREM Stage 2 sleep and spindles and, in fact, post-sleep motor performance correlated with sleep spindles. ${ }^{110}$ This hypothesis built on prior work noting that motor performance improvement correlated with NREM 
Stage 2 sleep ${ }^{97}$ - a stage rich in spindles. Several other studies have identified an increase in spindle density following a motor learning task, ${ }^{92,112,113}$ increased temporal grouping by cortical slow waves, ${ }^{114}$ and the existence of specific correlations between overnight performance improvements on motor tasks and spindle density, ${ }^{97,110,115}$ including over somatotopically relevant motor cortical areas. ${ }^{116}$ Selective spindle augmentation with transcranial alternating current stimulation (tACS) was shown to enhance motor memory consolidation. ${ }^{117}$

Integration of how REM sleep and NREM spindles interact to support motor learning and memory remains to be completed. It has been observed ${ }^{90}$ that most of the studies implicating a role for REM sleep have utilized a serial reaction time motor task with an implicit learning component, whereas studies implicating spindles generally use an explicit MST. It becomes tempting to then posit that REM sleep augments implicit motor learning whereas spindles augment explicit motor learning, but this has not been formally tested.

\section{Higher order cognition}

In spite of the frequency of use of the term sleep-dependent memory "consolidation", information encoded during wake states can be utilized in various ways that are not limited to the cementing of that information into long-term stores. This requires complex, discriminatory processing through which the brain triages relevant memories to be retained ${ }^{22,118}$ and, then, further goes on to qualitatively transform encoded information to facilitate future cognitive functioning - a concept referred to as complex associative memory processing. ${ }^{119}$

Sleep-dependent discriminatory memory processing has been described earlier with emotional memory, with which selective retention of emotional foreground - but not the background - elements occur. ${ }^{82}$ Furthermore, explicit "tagging" of an item as important or worth remembering during wakefulness modulates intentional memory processing and, therefore, determines the degree of subsequent recall. ${ }^{118,120}$ Sleep preferentially promoted retention of items cued to be remembered - but not those cued to be forgotten - across a daytime nap, with no differences in immediate recall. Selective sleep-dependent memory processing and recall has also been shown preferentially for items associated with an anticipated reward, ${ }^{121}$ perceived value, ${ }^{122}$ or with waking knowledge of future retesting. ${ }^{123}$

A study using fMRI has shown that, during the initial period of learning, there is increased hippocampal activity seen when presented with items cued as "to be remembered" than those marked as to be forgotten, which later translates to increased recall of the relevant items after a period of sleep. ${ }^{124}$ This role of sleep and the effectiveness of "cued" items is time sensitive. A delay in sleep onset $>24$ hours after encoding results in degradation of the cue and reduced recall. ${ }^{118}$ Moreover, there are data to suggest that selective memory processing is correlated with sleep spindles over the left superior parietal cortex, with increased density of spindles associated with appropriate remembering of cued items and forgetting of non-cued items. ${ }^{120}$

Beyond preferential determination of memory processing, complexities of sleep-dependent integration of new memories into existing paradigms have also been described. Single, newly learned memories can be integrated into preexisting schemas, allowing for enhanced assimilation and utility. The concept of "gist extraction" - whereby, when presented with multiple new memories, the brain identifies common features (or "gist") from them and incorporates them into a new schema - has been shown to be augmented by sleep. ${ }^{125}$ Sleep-dependent multi-item generalization may then allow for recognition of implicit patterns and creation of "overarching rules" that can then be extrapolated and applied to future experiences. ${ }^{118}$ In the transitive inference task, subjects are presented with pairs of objects that contain an embedded hierarchical structure (i.e., $\mathrm{A}>\mathrm{B}, \mathrm{B}>\mathrm{C}$, and $\mathrm{C}>\mathrm{D}$ ). When subjects are subsequently presented with novel pairs requiring secondorder inference (i.e., A and D), performance demonstrating an understanding of the hierarchical structure is enhanced after sleep, in comparison to wake states. ${ }^{126}$ A sleep-dependent enhancement of understanding of embedded structure is also demonstrated in the probabilistic learning weather-prediction task. During encoding, subjects are asked to predict outcomes (i.e., the weather) based on symbols, unaware that the symbols are linked with differing probabilities to the outcomes. Performance on such a task is enhanced after sleep ${ }^{127}$ and, across a nap, improved performance correlated with the amount of REM sleep. ${ }^{128}$ The understanding of a tacit structure can also be probed in implicit versions of serial reaction-time tests where subjects are asked whether they are able to explicitly identify the underlying pattern. In such studies, an objective understanding of the underlying structure after a period of sleep was associated with particular sleep features, including number of REM to NREM transitions ${ }^{129}$ and right-frontal fast-spindle activity. ${ }^{130}$ Finally, sleep may facilitate "predictive coding", ${ }^{131}$ or the ability of the brain to parse elements of pre-sleep experience that deviate from preformed schema - a feature that may rely, in particular, on REM sleep. 


\section{Targeted/cued memory reactivation}

Much of the experimental work understanding sleep's role in memory processing in human subjects stems from comparisons between sleep and equivalent periods of waking, or when particular physiologic features of sleep are either disrupted or imitated. However, there has been recent increasing interest in targeted memory reactivation (TMR), also known as cued memory reactivation - an interventional experimental technique that takes advantage of the brain's normal sleep-processing methods and offers additional insights into interactions between memory and sleep. Without any external influences, neuronal circuits activated by learning prior to sleep are thought to be spontaneously reactivated during sleep - primarily during SWS ${ }^{132}$ but also possibly during other NREM and REM stages. TMR involves triggering this reactivation of neuronal circuits through external means. Often, this involves exposing sleeping subjects to a sensory cue that was present during a prior encoding period. The effects of TMR vary based on the type of cue used, the sleep stage or stages in which the cueing occurred, and the type of memory reactivated.

Early studies employing TMR focused on REM sleep, perhaps influenced by work in rodents from the 1980 s indicating enhanced active avoidance conditioning memory when conditioned stimulus cues were reexpressed during REM sleep. ${ }^{133}$ A subsequent 1990 study tested performance improvement on a complex logic task in human subjects presented with the context of a ticking clock. Subjects reexposed to the ticking clock auditory cue during eye movements in REM sleep improved performance by $23 \%$ in comparison to a smaller $8 \%$ improvement across sleep when cues were absent during encoding and sleep. ${ }^{134}$ Additionally, performance on a Morse code task accompanied by an auditory sequence was significantly improved when the auditory stimulus was reintroduced during REM sleep. ${ }^{135}$ However, these studies did not provide electrophysiological evidence of undisrupted REM sleep and, as a result, the benefits of TMR could be theoretically attributed to the brief waking periods due to the stimulus cues. ${ }^{132}$

Two later influential studies implicated a role for TMR during NREM sleep in forms of two-dimensional (2D) spatial memory. In one study, images of objects on a screen were paired with sounds during learning and, after a daytime nap, subjects were asked to place the object in the location on the screen it had been viewed. Location accuracy was significantly enhanced when the paired sounds were played during NREM sleep, and the degree of improvement correlated with EEG amplitude responses to the auditory stimuli during sleep. ${ }^{136}$ In the other study by Rasch et al, subjects learned an object-location task where they were asked to visually learn the locations of 15 unique card pairs presented on a computer screen. ${ }^{137}$ While performing the task, subjects were exposed to a rose scent, to which they were reexposed during SWS. Most notably, in the 30-second intervals during SWS where puffs of rose scent were administered, hippocampal regions showed significant activity on fMRI scans, suggesting that the rose scent was externally reactivating memories of the object-location task. Sleep was not disrupted according to EEG recordings, and participants exposed to the odor cue during SWS showed distinctly improved performance when compared with the vehicle group. Odor exposure during REM sleep or wake following encoding did not impart such a benefit. Subsequent research on language learning supported this work, where participants fluent in the German language were presented with Dutch words presented over auditory speakers and were asked to verbally translate the word in German. ${ }^{138}$ The foreign words were verbally presented during NREM sleep, resulting in improved subsequent German translation.

Of note, in Rasch et al's study, an explicit finger-tapping MST was also tested where odor was presented during presleep encoding. Subsequent reexposure to the odor during SWS, REM sleep, or wake states did not improve performance in comparison to exposure to vehicle. One explanation for this may be that the finger-tapping sequence task may not be as easily associated with olfactory cues as auditory cues. This suggests that different sensory cues may be better suited for certain memory tasks than others. This possibility is supported by studies in which motor learning tasks were cued with auditory stimuli. In a task resembling the game Guitar Hero, participants were taught to play two different 12-item piano sequences of four notes. ${ }^{139}$ The sequence cued during SWS in a subsequent afternoon nap showed greater performance improvement than the sequence not cued. In a similar study, participants learned a piano sequence, but only half of the sequence was replayed during sleep. ${ }^{140}$ Participants only showed improvement on the half that was cued during SWS, adding to the idea of TMR benefiting motor memory.

The above results argue predominantly for a positive effect of cue reexposure during SWS on declarative and motor memories; however, there exists evidence for interesting interactions between SWS and REM sleep when TMR is employed. When auditory cues for novel vocabulary words learned before sleep were presented exclusively during SWS, post-sleep performance was significantly influenced by REM sleep duration such that participants with longer REM duration showed a significant benefit for cued - relative to uncued 
- words, whereas participants who obtained little or no REM demonstrated a significant effect in the opposite direction. ${ }^{141}$ When auditory tones associated with a motor serial reaction time test were replayed exclusively during SWS, increased activity in the bilateral caudate nucleus and hippocampus was associated with time in SWS, whereas increased cerebellar and cortical motor activity was related to time in REM sleep when brain fMRI was used during post-sleep performance of the motor task. ${ }^{142}$ This suggests that memory processing is differentially modulated by REM and SWS. Interestingly, it has been suggested that TMR during REM sleep may be involved in memory generalization, as auditory cues for previously learned faces delivered during REM sleep enhanced not only subsequent accurate recollections but also false recognitions. ${ }^{143}$

An interesting application of TMR that also highlights SWS and REM sleep interactions is in reducing stereotypes and social biases. In a study aimed to reduce social biases, subjects were taught to match faces to a word meant to counter stereotypes associated with the gender or racial identity of the face. ${ }^{144}$ When the match was correctly made, a distinct sound was played for the type of match correctly made: either a counter-gender-bias match or counter-race-bias match. During SWS, one of the auditory cues was played. Implicit biases were unchanged for uncued biases, but significantly reduced for cued biases. This bias reduction through TMR was preserved a week later, and larger bias reduction over this interval for the cued - compared to the uncued - bias was positively associated with the product of SWS and REM sleep duration (i.e., $\mathrm{SWS} \times \mathrm{REM}$ ).

\section{OSA and memory}

OSA is a sleep disorder characterized by repetitive upper airway collapse that leads to cycles of intermittent hypoxia, sleep fragmentation, and/or intrathoracic pressure swings that impact cerebrospinal fluid flow and possible clearance of neuronal metabolites in the interstitial space. ${ }^{145}$ Per current American Academy of Sleep Medicine scoring criteria, an apnea is scored when there is complete lack of measurable breathing airflow lasting $\geq 10$ seconds. A hypopnea is scored when there is a reduction in the amplitude of breathing by $30 \%$ or more for $\geq 10$ seconds accompanied by $\geq 3 \%$ decline in blood oxygen saturation or an arousal indicated by a sudden increase in the cortical EEG frequency or by sudden increase in the motor tone in the chin or anterior tibealis by electromyography. The AHI3a reflects the sum of all apneas and hypopneas divided by the total sleep time in hours. An older definition of hypopnea (still used by the Centers for Medicare and Medicaid Services) is a reduction in the amplitude of breathing by $30 \%$ or more for $\geq 10$ seconds with $\geq 4 \%$ decline in blood oxygen saturation, irrespective of the presence of an arousal. The AHI using this definition of hypopnea is abbreviated AHI4\% in our work.

These repetitive upper airway phenomena likely affect the amount of, and orderly progression through, specific stages of sleep. As noted at the beginning of this review, memory is broadly divided into processes of encoding, processing/consolidation, and retrieval. Whereas sleep loss and associated sleepiness can impact encoding, the current understanding of the neural mechanisms underlying memory formation primarily implicates a role for sleep in the processing/consolidation phase. It would, therefore, stand to reason that OSA is most likely to have a significant impact on precisely this processing/consolidation phase. Doing so would require testing task performance before and after a period of sleep - ideally, a period of sleep containing either apnea or no apnea within the same individual. However, by far the vast majority of research on sleep apnea and memory employs memory tasks for which there is little, if any, opportunity for processing/consolidation and, when there is, it is within a daytime period of normal wakefulness. As a consequence, opportunities to test what are likely to be the strongest effects of OSA on memory are missed.

In the small number of studies in which effects of OSA on memory processing/consolidation have been examined, deleterious consequences of OSA have been seen rather consistently. As highlighted in the spatial navigational memory section earlier, based on the suspected role for REM sleep in memory spatial navigational memory processing, OSA was induced exclusively during REM through CPAP withdrawal in subjects with known severe OSA who use CPAP regularly. ${ }^{66} \mathrm{~A} 3 \mathrm{D}$ computer-generated virtual navigation task was undertaken both before and after a night of polysomnographically recorded sleep. When sleep was normally consolidated and CPAP was held at the therapeutic level, there was an overnight performance improvement evidenced by a $31 \%$ reduction in maze completion time. In comparison, when OSA was induced in REM in the same subjects, this benefit was abolished and it took subjects 5\% longer to complete the maze, in spite of no changes in morning psychomotor vigilance.

The majority of other studies that have examined an effect of OSA on memory processing/consolidation have done so in the motor memory domain. In two related papers, subjects with and without OSA were trained on a finger-tapping explicit MST before and after sleep. In the earlier study, ${ }^{146}$ the 
average AHI terminating in a $\geq 3 \%$ drop in oxygen or arousal (AHI3a) in OSA subjects was 17 /hour, suggesting overall very mild disease. Nonetheless, control subjects without OSA demonstrated a greater offline immediate improvement (last three training trials in the evening vs. the first three test trials in the morning) and offline plateau improvement (last six training trials in the evening vs. the last six test trials in the morning) in motor performance. Furthermore, across all subjects, the strongest measured correlate of plateau improvement was the arousal index, but no correlation was observed with the oxygen-saturation nadir, suggesting that perhaps a minimum amount of sleep continuity is needed for memory consolidation - a process that may be interrupted by even mild OSA. In the latter study, ${ }^{147}$ OSA severity was also mild with an average AHI3a of 25/hour and, again, significant offline gains were observed in control subjects, who improved $15 \%$ when comparing the last three training trials in the evening vs. the first three test trials in the morning, but not in OSA subjects, who improved just $2 \%$ on the same metric. Of note, in this study, OSA subjects had a significantly lower speed during the last three pre-sleep trials compared to control subjects, suggesting an encoding deficit cannot be ruled out. Notably, this effect may be mediated by age, ${ }^{148}$ and OSA severity is known to increase with age. ${ }^{9}$ Interestingly, when CPAP was applied for the first time in subjects with OSA, significant offline gains in motor performance on this finger-tapping MST were not observed. ${ }^{149}$ However, one potential confound to this observation is that subjects with OSA who were about to receive CPAP for the first time appeared to have significantly lower evening performance in either the last three or six trials, although this was not directly reported. As such, a deficit in initial pre-sleep encoding cannot be ruled out, although subjects receiving CPAP for the first time reported improved subjective morning refreshment on average. This highlights a significant limitation of assessing performance on the very first night of CPAP treatment, and reassessment after subjects have been stably using CPAP for a significant duration might aid interpretation. Outside of the finger-tapping MST, significant overnight offline improvements were observed in multiple measures of a motor mirror-tracing task in control subjects that were significantly attenuated in subjects with mild OSA (average AHI3a 20/hour), ${ }^{150}$ and, in a serial reaction time test, a greater fraction of controls showed offline gains than severe OSA subjects (mean AHI3a 53/hour). ${ }^{151}$ These OSA subjects were significantly slower at this serial reaction time task during pre-sleep training - again suggesting a possible encoding effect.
In non-motor memory tasks, verbal declarative memory was tested simultaneously with mirror tracing in the earlier study looking at mild OSA subjects. ${ }^{150}$ OSA subjects showed a significantly reduced verbal retention rate after sleep compared to healthy controls. Children with OSA trained on an evening picture-based visual memory task displayed both slower pre-sleep encoding rates and poorer overnight retention than healthy control children. ${ }^{152}$ Likewise, children with OSA displayed poorer sleep-dependent consolidation on a spatial 2D card-matching task than children without OSA. ${ }^{153}$ Notably, this study also examined a group of children with primary snoring and no OSA who performed similarly to children with no OSA and no snoring.

OSA would appear to most likely have a deleterious effect on sleep-dependent memory processing, but it is possible that OSA also has effects on encoding or retrieval. The vast majority of studies investigating a role for sleep apnea on memory have exclusively employed daytime tests with little opportunity for processing/consolidation. Possibly as a consequence of this, the reported effects of OSA on memory have been quite varied. A recent review nicely posited some of the potential etiologies of this heterogeneity, including differences in subject age, cognitive reserve, genetic factors known to influence cognition such as the ApoE genotype, generation of sleepiness in response to OSA, duration of OSA before evaluation, and OSA pathogenesis. ${ }^{154}$ Additionally, heterogeneity on memory effects may be influenced by the extent to which the presence of OSA is associated with other health comorbidities that may impact cognition, such as cerebrovascular disease and insulin resistance. ${ }^{155,156}$ Across several meta-analyses of OSA and cognition, the strongest negative effects of OSA have been on executive function and attention, ${ }^{157,158}$ which could then have differential effects on daytime memory encoding or retrieval, depending on the task. A meta-analysis specifically focusing on OSA and memory per se noted specific deficits in forms of episodic memory, including verbal episodic memory and visuospatial episodic memory. ${ }^{159}$ This latter observation was echoed in another meta-analysis where deficits in visuospatial memory were noted in OSA, but not in short-term sleep deprivation, insomnia, or chronic obstructive pulmonary disease (COPD) - disorders that have some overlapping pathophysiology with OSA. ${ }^{160}$

A role for OSA in impairing memory would be strengthened by establishing some form of dose dependence, such as parametric manipulation of variables including, but not limited to, the AHI - the variable typically used to define severity, as well as establishing response to treatment. Two 
large, randomized, controlled trials of OSA treatment that focused on cognitive outcomes include the Apnea PositivePressure Long-Term Efficacy Study (APPLES) ${ }^{161}$ in adults and the Childhood Adenotonsillectomy Trial (CHAT) ${ }^{162}$ study in children. In APPLES, subjects with newly diagnosed OSA were randomized to CPAP vs. sham and assessed at subsequent 2-month and 6-month time points. Primary outcomes included performance on tests of three different cognitive domains: 1) Pathfinder Number Test-Total Time (i.e., computer-based Trails A) for attention and psychomotor function, 2) Buschke Selective Reminding Test-Sum Recall for verbal memory, and 3) Sustained Working Memory TestOverall Mid-Day Index for executive function. Although, as a whole, there were no differences in any of these primary outcomes at either time point, sub-analyses showed that those with the most severe OSA randomized to CPAP treatment vs. sham had better executive function at the 2-month, but not 6-month, time point, and those with baseline Epworth Sleepiness Scores $>10$ randomized to CPAP treatment vs. sham had better executive function at the 6-month time point. In CHAT, children aged 5-9 with OSA were randomized to either early adenotonsillectomy or watchful waiting for a period of 7 months. Although children receiving OSA treatment via early adenotonsillectomy had confirmed polysomnographic improvement in OSA severity and were observed to have improvements in behavioral and quality-of-life measures vs. those randomized to watchful waiting, no significant differences between groups were observed in the primary cognitive outcome testing for attention and executive function.

It is generally assumed that much of the pathophysiology of OSA is reversed with chronic treatment. However, there is a school of thought which argues that there are effects due to irreversible apnea/hypoxia-induced damage that would be reflected in an absence of memory improvement even after treatment. For example, subjects with mild to moderate OSA treated with CPAP for 3 months continued to show some impairments in psychomotor vigilance and working memory compared to subjects of similar age and demography without OSA. ${ }^{163}$ It is possible that the lack of pronounced effect of OSA treatment on cognitive outcomes in the APPLES and CHAT studies is consistent with this idea; however, there are a number of alternative possibilities, including the criticism that the combination of tasks and general high functioning of the enrolled subjects created a ceiling effect, ${ }^{164,165}$ making it difficult to appreciate benefits of OSA treatment. It is important to additionally bear in mind that specific memory testing was but a small part of these studies and, again, any memory testing was completed during daytime testing sessions without opportunity for sleep-dependent processing to occur. Table 1 summarizes key papers implicating both normal sleep and sleep apnea in various forms of memory processing.

\section{Conclusion}

The available evidence suggests that sleep is indeed important for forms of offline memory processing or consolidation, but perhaps does so in ways that are specific to particular types of memories, revealed by specific types of memory tests. Neurophysiological properties of sleep such as slow oscillations, spindles, and REM sleep appear to be more important for some types of memory other others, and how these features interact to bring about the phenomenon of offline gains in performance is only beginning to be elucidated. OSA is a common disorder, well poised to specifically affect the offline processing or consolidation of memories or the augmentation of memory through targeted memory reactivation; however, in fact, these possibilities have been tested only quite minimally. Furthermore, although it seems intuitive that OSA could have the capacity to interrupt slow oscillations, spindles, or REM sleep, emerging evidence

Table I Key references for the role of sleep and obstructive sleep apnea (OSA) on different memory types

\begin{tabular}{|c|c|}
\hline Declarative memory & $\begin{array}{l}\text { Plihal and Born; }{ }^{23} \text { Mander et al; } ; 2 \text { Van Der Werf et al,;2 Marshall et al; }{ }^{30} \text { Westerberg et al; }{ }^{31} \text { Leminen et al; }{ }^{32} \text { Yoo } \\
\text { et al } ;^{38} \text { Van Der Werf et al, }{ }^{39} \text { Gais et al }{ }^{43}\end{array}$ \\
\hline Spatial memory & $\begin{array}{l}\text { Orban et al }{ }^{50} \text { Nguyen et al; } ;{ }^{51} \text { Ferrara et al, }{ }^{52} \text { Wamsley et al } ;{ }^{53} \text { Wamsley et al } ;{ }^{54} \text { Varga et al } ; ;^{55} \text { Peigneux et al } ;{ }^{56} \\
\text { Varga et al; } ; 6 \text { Rauchs et al }{ }^{70}\end{array}$ \\
\hline Emotional memory & 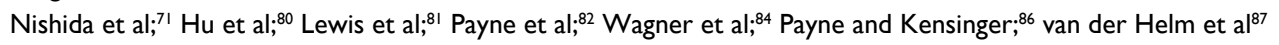 \\
\hline Motor memory & $\begin{array}{l}\text { Albouy et al; }{ }^{88} \text { Debas et al; }{ }^{94} \text { Fischer et al; }{ }^{96} \text { Walker et al; } ;{ }^{77} \text { Kuriyama et al; } ; 9 \text { Cohen et al; }{ }^{102} \text { Nitsche et al; }{ }^{107} \\
\text { Rasch et al; }{ }^{110} \text { Nishida and Walker; } ;{ }^{13} \text { Johnson et al; }{ }^{116} \text { Lustenberger et al }{ }^{117}\end{array}$ \\
\hline Higher order cognition & $\begin{array}{l}\text { Saletin et al; }{ }^{120} \text { Fischer and Born; }{ }^{121} \text { van Dongen et al; }{ }^{123} \text { Payne et al; }{ }^{125} \text { Ellenbogen et al; } ;{ }^{126} \text { Djonlagic et al; }{ }^{127} \\
\text { Barsky et al; }{ }^{128} \text { Yordanova et al }{ }^{130}\end{array}$ \\
\hline Targeted memory reactivation & Rudoy et al; ${ }^{136}$ Rasch et al; ${ }^{137}$ Antony et al; ${ }^{139}$ Schönauer et al; ${ }^{140}$ Cousins et al; ${ }^{142}$ Sterpenich et al; ${ }^{143} \mathrm{Hu}$ et al ${ }^{144}$ \\
\hline OSA effects on memory & $\begin{array}{l}\text { Djonlagic et al; }{ }^{146} \text { Landry et al; }{ }^{147} \text { Kloepfer et al; }{ }^{150} \text { Csabi et al; }{ }^{151} \text { Kheirandish-Gozal et al; }{ }^{152} \text { Maski et al; }{ }^{153} \text { Kushida } \\
\text { et al; }{ }^{161} \text { Marcus et al }{ }^{162}\end{array}$ \\
\hline
\end{tabular}




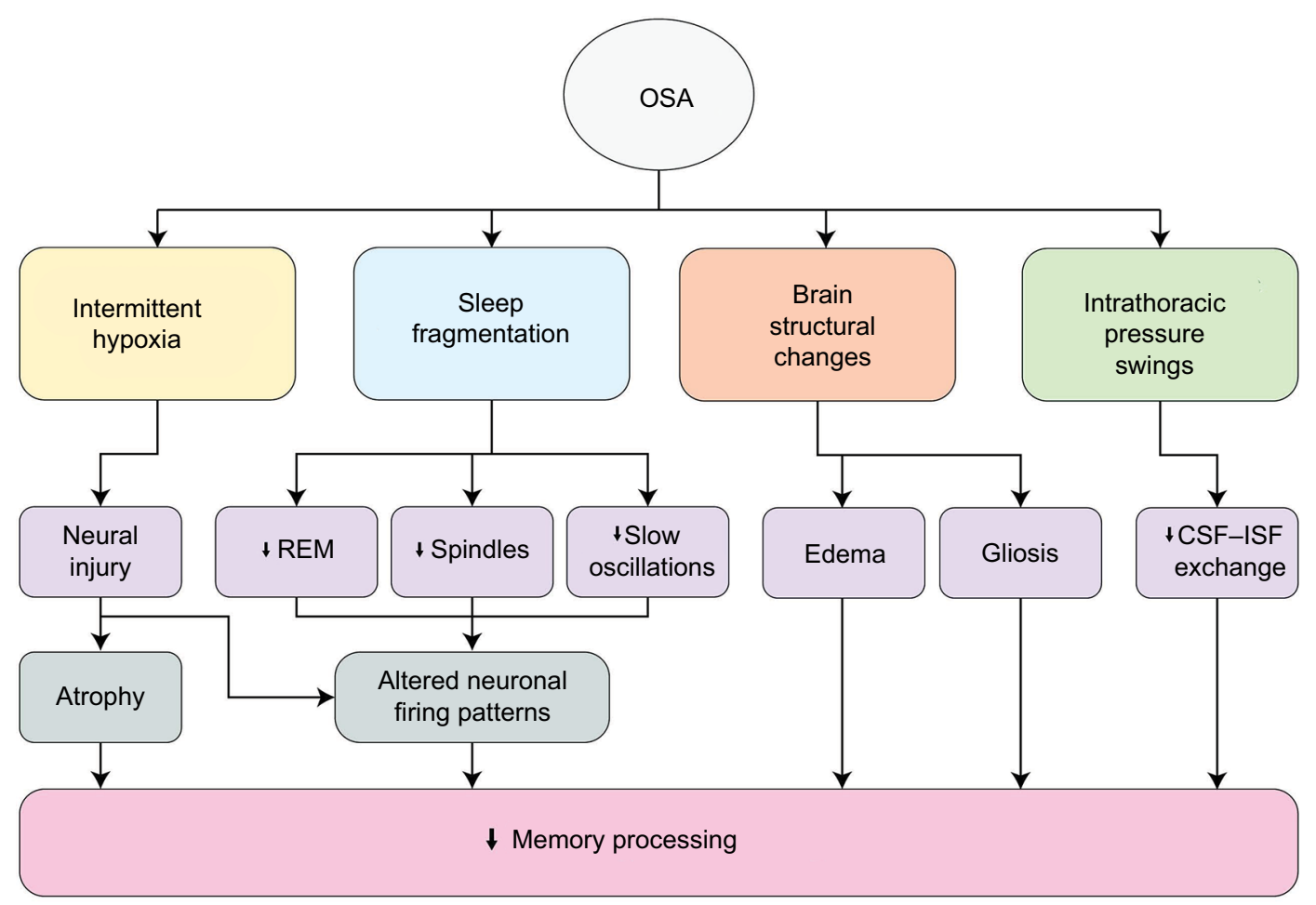

Figure I Multiple mechanisms by which OSA can impact memory processing.

Abbreviations: CSF, cerebrospinal fluid; ISF, interstitial fluid; OSA, obstructive sleep apnea; REM, rapid eye movement.

suggests it does so in ways that might be nonlinear. Fundamental questions remain about the potentially unique contributions of intermittent hypoxia and sleep fragmentation on memory, let alone intrathoracic pressure swings and additional recently identified brain structural consequences of OSA such as gray matter edema, ${ }^{166}$ gliosis, and other microstructural changes evidenced by mean diffusivity/fractional anisotropy changes ${ }^{167,168}$ (Figure 1). Thus, although we have some answers about the impact of OSA on memory, clearly multiple avenues of investigation remain wide open.

\section{Acknowledgments}

This work was supported by grants from: NIH/NIA/ NHLBI R01AG056682, R21AG055002, R01HL118624, R01AG022374, and P30AG008051; the American Sleep Medicine Foundation Junior Faculty Award, the American Thoracic Society Foundation, and the Friedman Brain Institute.

\section{Disclosure}

The authors report no conflicts of interest in this work.

\section{References}

1. Muller GE, Pilzecker A. Experimentelle beitrage zur lehre von gedächtnis [experimental contributions to the theory of memory]. Z Psychol. 1900;1:1-300. German.
2. Jenkins JG, Dallenbach KM. Obliviscence during sleep and waking. Am J Psychol. 1924;35(4):605-612.

3. Rasch B, Born J. About sleep's role in memory. Physiol Rev. 2013;93(2): 681-766.

4. Diekelmann S, Wilhelm I, Born J. The whats and whens of sleep-dependent memory consolidation. Sleep Med Rev. 2009;13(5):309-321.

5. Olesen J, Gustavsson A, Svensson M, Wittchen HU, Jönsson B; CDBE2010 study group; European Brain Council. The economic cost of brain disorders in Europe. Eur J Neurol. 2012;19(1):155-162.

6. Institute of Medicine Committee on Sleep M, Research. The National Academies Collection: reports funded by National Institutes of Health. In: Colten HR, Altevogt BM, editors. Sleep Disorders and Sleep Deprivation: an Unmet Public Health Problem. Washington, DC: National Academies Press (US) National Academy of Sciences; 2006.

7. Young T, Palta M, Dempsey J, Skatrud J, Weber S, Badr S. The occurrence of sleep-disordered breathing among middle-aged adults. NEngl J Med. 1993;328(17):1230-1235.

8. Heinzer R, Vat S, Marques-Vidal P, et al. Prevalence of sleep-disordered breathing in the general population: the HypnoLaus study. Lancet Respir Med. 2015;3(4):310-318.

9. Sharma RA, Varga AW, Bubu OM, et al. Obstructive sleep apnea severity affects amyloid burden in cognitively normal elderly: a longitudinal study. Am J Respir Crit Care Med. 2018;197(7):933-943.

10. Skaggs WE, McNaughton BL. Replay of neuronal firing sequences in rat hippocampus during sleep following spatial experience. Science. 1996;271(5257):1870-1873.

11. Wilson MA, McNaughton BL. Reactivation of hippocampal ensemble memories during sleep. Science. 1994;265(5172):676-679.

12. Qin YL, McNaughton BL, Skaggs WE, Barnes CA. Memory reprocessing in corticocortical and hippocampocortical neuronal ensembles. Philos Trans R Soc Lond B Biol Sci. 1997;352(1360):1525-1533.

13. Ji D, Wilson MA. Coordinated memory replay in the visual cortex and hippocampus during sleep. Nat Neurosci. 2007;10(1):100-107.

14. Diekelmann S, Born J. The memory function of sleep. Nat Rev Neurosci. 2010;11(2):114-126. 
15. Cellini N. Memory consolidation in sleep disorders. Sleep Med Rev. 2017;35:101-112.

16. Buzsáki G. The hippocampo-neocortical dialogue. Cereb Cortex. 1996;6(2):81-92.

17. McClelland JL, McNaughton BL, O'Reilly RC. Why there are complementary learning systems in the hippocampus and neocortex: insights from the successes and failures of connectionist models of learning and memory. Psychol Rev. 1995;102(3):419-457.

18. Siapas AG, Wilson MA. Coordinated interactions between hippocampal ripples and cortical spindles during slow-wave sleep. Neuron. 1998;21(5):1123-1128.

19. Sirota A, Csicsvari J, Buhl D, Buzsáki G. Communication between neocortex and hippocampus during sleep in rodents. Proc Natl Acad Sci U S A. 2003;100(4):2065-2069.

20. Jiang X, Shamie I, K Doyle W, et al. Replay of large-scale spatiotemporal patterns from waking during subsequent NREM sleep in human cortex. Sci Rep. 2017;7(1):17380.

21. Takashima A, Petersson KM, Rutters F, et al. Declarative memory consolidation in humans: a prospective functional magnetic resonance imaging study. Proc Natl Acad Sci U SA. 2006;103(3):756-761.

22. Stickgold R. Parsing the role of sleep in memory processing. Curr Opin Neurobiol. 2013;23(5):847-853.

23. Plihal W, Born J. Effects of early and late nocturnal sleep on declarative and procedural memory. J Cogn Neurosci. 1997;9(4):534-547.

24. Plihal W, Born J. Effects of early and late nocturnal sleep on priming and spatial memory. Psychophysiology. 1999;36(5):571-582.

25. Barrett TR, Ekstrand BR. Effect of sleep on memory. 3. Controlling for time-of-day effects. $J$ Exp Psychol. 1972;93:321-327.

26. Yaroush R, Sullivan MJ, Ekstrand BR. Effect of sleep on memory. II. Differential effect of the first and second half of the night. $J$ Exp Psychol. 1971;88(3):361-366.

27. Mander BA, Rao V, Lu B, et al. Prefrontal atrophy, disrupted NREM slow waves and impaired hippocampal-dependent memory in aging. Nat Neurosci. 2013;16(3):357-364.

28. Van Der WerfYD, Altena E, Vis JC, Koene T, Van Someren EJ. Reduction of nocturnal slow-wave activity affects daytime vigilance lapses and memory encoding but not reaction time or implicit learning. Prog Brain Res. 2011;193:245-255.

29. Marshall L, Mölle M, Hallschmid M, Born J. Transcranial direct current stimulation during sleep improves declarative memory. J Neurosci. 2004;24(44):9985-9992.

30. Marshall L, Helgadóttir H, Mölle M, Born J. Boosting slow oscillations during sleep potentiates memory. Nature. 2006;444(7119):610-613.

31. Westerberg CE, Florczak SM, Weintraub S, et al. Memory improvement via slow-oscillatory stimulation during sleep in older adults. Neurobiol Aging. 2015;36(9):2577-2586.

32. Leminen MM, Virkkala J, Saure E, et al. Enhanced memory consolidation via automatic sound stimulation during non-REM sleep. Sleep. 2017;40(3).

33. Vienne J, Lecciso G, Constantinescu I, et al. Differential effects of sodium oxybate and baclofen on EEG, sleep, neurobehavioral performance, and memory. Sleep. 2012;35(8):1071-1083.

34. Feld GB, Wilhelm I, Ma Y, et al. Slow wave sleep induced by GABA agonist tiagabine fails to benefit memory consolidation. Sleep. 2013;36(9):1317-1326.

35. Alger SE, Lau H, Fishbein W. Delayed onset of a daytime nap facilitates retention of declarative memory. PLoS One. 2010;5(8):e12131.

36. Schönauer M, Pawlizki A, Köck C, Gais S. Exploring the effect of sleep and reduced interference on different forms of declarative memory. Sleep. 2014;37(12):1995-2007.

37. McDermott CM, LaHoste GJ, Chen C, Musto A, Bazan NG, Magee JC. Sleep deprivation causes behavioral, synaptic, and membrane excitability alterations in hippocampal neurons. $J$ Neurosci. 2003;23(29):9687-9695.

38. Yoo SS, Hu PT, Gujar N, Jolesz FA, Walker MP. A deficit in the ability to form new human memories without sleep. Nat Neurosci. 2007;10(3):385-392.
39. Van Der WerfYD, Altena E, Schoonheim MM, et al. Sleep benefits subsequent hippocampal functioning. Nat Neurosci. 2009;12(2):122-123.

40. Mander BA, Santhanam S, Saletin JM, Walker MP. Wake deterioration and sleep restoration of human learning. Curr Biol. 2011;21(5): R183-R184.

41. Tononi G, Cirelli C. Sleep function and synaptic homeostasis. Sleep Med Rev. 2006;10(1):49-62.

42. Steriade M. Impact of network activities on neuronal properties in corticothalamic systems. J Neurophysiol. 2001;86(1):1-39.

43. Gais S, Mölle M, Helms K, Born J. Learning-dependent increases in sleep spindle density. $J$ Neurosci. 2002;22(15):6830-6834.

44. Wilhelm I, Diekelmann S, Born J. Sleep in children improves memory performance on declarative but not procedural tasks. Learn Mem. 2008;15(5):373-377.

45. Ferri R, Drago V, Aricò D, et al. The effects of experimental sleep fragmentation on cognitive processing. Sleep Med. 2010;11(4):378-385.

46. Kunz L, Schröder TN, Lee H, et al. Reduced grid-cell-like representations in adults at genetic risk for Alzheimer's disease. Science. 2015;350(6259):430-433.

47. Buzsáki G, Moser EI. Memory, navigation and theta rhythm in the hippocampal-entorhinal system. Nat Neurosci. 2013;16(2):130-138.

48. Iaria G, Petrides M, Dagher A, Pike B, Bohbot VD. Cognitive strategies dependent on the hippocampus and caudate nucleus in human navigation: variability and change with practice. $J$ Neurosci. 2003;23(13):5945-5952.

49. Chersi F, Burgess N. The cognitive architecture of spatial navigation: hippocampal and striatal contributions. Neuron. 2015;88(1):64-77.

50. Orban P, Rauchs G, Balteau E, et al. Sleep after spatial learning promotes covert reorganization of brain activity. Proc Natl Acad Sci U S A. 2006;103(18):7124-7129.

51. Nguyen ND, Tucker MA, Stickgold R, Wamsley EJ. Overnight sleep enhances hippocampus-dependent aspects of spatial memory. Sleep. 2013;36(7):1051-1057.

52. Ferrara M, Iaria G, Tempesta D, et al. Sleep to find your way: the role of sleep in the consolidation of memory for navigation in humans. Hippocampus. 2008;18(8):844-851.

53. Wamsley EJ, Tucker M, Payne JD, Benavides JA, Stickgold R. Dreaming of a learning task is associated with enhanced sleep-dependent memory consolidation. Curr Biol. 2010;20(9):850-855.

54. Wamsley EJ, Tucker MA, Payne JD, Stickgold R. A brief nap is beneficial for human route-learning: the role of navigation experience and EEG spectral power. Learn Mem. 2010;17(7):332-336.

55. Varga AW, Ducca EL, Kishi A, et al. Effects of aging on slow-wave sleep dynamics and human spatial navigational memory consolidation. Neurobiol Aging. 2016;42:142-149.

56. Peigneux P, Laureys S, Fuchs $\mathrm{S}$, et al. Are spatial memories strengthened in the human hippocampus during slow wave sleep? Neuron. 2004;44(3):535-545.

57. Youngblood BD, Zhou J, Smagin GN, Ryan DH, Harris RB. Sleep deprivation by the "flower pot" technique and spatial reference memory. Physiol Behav. 1997;61(2):249-256.

58. Smith C, Rose GM. Evidence for a paradoxical sleep window for place learning in the Morris water maze. Physiol Behav. 1996;59(1): 93-97.

59. Beaulieu I, Godbout R. Spatial learning on the Morris Water Maze Test after a short-term paradoxical sleep deprivation in the rat. Brain Cogn. 2000;43(1-3):27-31.

60. Li S, Tian Y, Ding Y, Jin X, Yan C, Shen X. The effects of rapid eye movement sleep deprivation and recovery on spatial reference memory of young rats. Learn Behav. 2009;37(3):246-253.

61. Yang RH, Hu SJ, Wang Y, Zhang WB, Luo WJ, Chen JY. Paradoxical sleep deprivation impairs spatial learning and affects membrane excitability and mitochondrial protein in the hippocampus. Brain Res. 2008; 1230:224-232.

62. Wang GP, Huang LQ, Wu HJ, Zhang L, You ZD, Zhao ZX. Calcineurin contributes to spatial memory impairment induced by rapid eye movement sleep deprivation. Neuroreport. 2009;20(13):1172-1176. 
63. Smith CT, Conway JM, Rose GM. Brief paradoxical sleep deprivation impairs reference, but not working, memory in the radial arm maze task. Neurobiol Learn Mem. 1998;69(2):211-217.

64. Bjorness TE, Riley BT, Tysor MK, Poe GR. REM restriction persistently alters strategy used to solve a spatial task. Learn Mem. 2005;12(3):352-359.

65. Boyce R, Glasgow SD, Williams S, Adamantidis A. Causal evidence for the role of REM sleep theta rhythm in contextual memory consolidation. Science. 2016;352(6287):812-816.

66. Varga AW, Kishi A, Mantua J, et al. Apnea-induced rapid eye movement sleep disruption impairs human spatial navigational memory. J Neurosci. 2014;34(44):14571-14577.

67. Braun AR, Balkin TJ, Wesenten NJ, et al. Regional cerebral blood flow throughout the sleep-wake cycle. An H2(15)O PET study. Brain. 1997;120(Pt 7):1173-1197.

68. Urbain N, Gervasoni D, Soulière F, et al. Unrelated course of subthalamic nucleus and globus pallidus neuronal activities across vigilance states in the rat. Eur J Neurosci. 2000;12(9):3361-3374.

69. Sastre JP, Buda C, Lin JS, Jouvet M. Differential c-fos expression in the rhinencephalon and striatum after enhanced sleep-wake states in the cat. Eur J Neurosci. 2000;12(4):1397-1410.

70. Rauchs G, Orban P, Schmidt C, et al. Sleep modulates the neural substrates of both spatial and contextual memory consolidation. PLoS One. 2008;3(8):e2949.

71. Nishida M, Pearsall J, Buckner RL, Walker MP. REM sleep, prefrontal theta, and the consolidation of human emotional memory. Cereb Cortex. 2009;19(5):1158-1166.

72. Wagner U, Gais S, Haider H, Verleger R, Born J. Sleep inspires insight. Nature. 2004;427(6972):352-355.

73. McGaugh JL. The amygdala modulates the consolidation of memories of emotionally arousing experiences. Ann Rev Neurosci. 2004;27(1):1-28

74. van der Helm E, Walker MP. Sleep and emotional memory processing. Sleep Med Clin. 2011;6(1):31-43.

75. Strange BA, Hurlemann R, Dolan RJ. An emotion-induced retrograde amnesia in humans is amygdala- and beta-adrenergic-dependent. Proc Natl Acad Sci U S A. 2003;100(23):13626-13631.

76. Cahill L, Prins B, Weber M, McGaugh JL. Beta-adrenergic activation and memory for emotional events. Nature. 1994;371(6499):702-704

77. Amaral DG, Behniea H, Kelly JL. Topographic organization of projections from the amygdala to the visual cortex in the macaque monkey. Neuroscience. 2003;118(4):1099-1120.

78. Phelps EA. Human emotion and memory: interactions of the amygdala and hippocampal complex. Curr Opin Neurobiol. 2004;14(2):198-202.

79. Payne JD, Kensinger EA. Sleep's role in the consolidation of emotional episodic memories. CurrDir Psychol Sci. 2010;19(5):290-295.

80. Hu P, Stylos-Allan M, Walker MP. Sleep facilitates consolidation of emotional declarative memory. Psychol Sci. 2006;17(10):891-898.

81. Lewis PA, Cairney S, Manning L, Critchley HD. The impact of overnight consolidation upon memory for emotional and neutral encoding contexts. Neuropsychologia. 2011;49(9):2619-2629.

82. Payne JD, Stickgold R, Swanberg K, Kensinger EA. Sleep preferentially enhances memory for emotional components of scenes. Psychol Sci. 2008;19(8):781-788.

83. Walker MP, van der Helm E. Overnight therapy? The role of sleep in emotional brain processing. Psychol Bull. 2009;135(5):731-748.

84. Wagner U, Gais S, Born J. Emotional memory formation is enhanced across sleep intervals with high amounts of rapid eye movement sleep. Learn Mem. 2001;8(2):112-119.

85. Goldstein AN, Walker MP. The role of sleep in emotional brain function. Annu Rev Clin Psychol. 2014;10:679-708.

86. Payne JD, Kensinger EA. Sleep leads to changes in the emotional memory trace: evidence from FMRI. J Cogn Neurosci. 2011;23(6):1285-1297.

87. van der Helm E, Yao J, Dutt S, Rao V, Saletin JM, Walker MP. REM sleep depotentiates amygdala activity to previous emotional experiences. Curr Biol. 2011;21(23):2029-2032.
88. Albouy G, King BR, Maquet P, Doyon J. Hippocampus and striatum: dynamics and interaction during acquisition and sleeprelated motor sequence memory consolidation. Hippocampus. 2013;23(11):985-1004.

89. Albouy G, Sterpenich V, Vandewalle G, et al. Interaction between hippocampal and striatal systems predicts subsequent consolidation of motor sequence memory. PLoS One. 2013;8(3):e59490.

90. King BR, Hoedlmoser K, Hirschauer F, Dolfen N, Albouy G. Sleeping on the motor engram: the multifaceted nature of sleep-related motor memory consolidation. Neurosci Biobehav Rev. 2017;80:1-22.

91. Smith C, MacNeill C. Impaired motor memory for a pursuit rotor task following Stage 2 sleep loss in college students. J Sleep Res. 1994;3(4):206-213.

92. Fogel SM, Smith CT. Learning-dependent changes in sleep spindles and Stage 2 sleep. J Sleep Res. 2006;15(3):250-255.

93. Mantua J, Baran B, Spencer RM. Sleep benefits consolidation of visuo-motor adaptation learning in older adults. Exp Brain Res. 2016;234(2):587-595.

94. Debas K, Carrier J, Orban P, et al. Brain plasticity related to the consolidation of motor sequence learning and motor adaptation. Proc Natl Acad Sci U S A. 2010;107(41):17839-17844.

95. Albouy G, Vandewalle G, Sterpenich V, et al. Sleep stabilizes visuomotor adaptation memory: a functional magnetic resonance imaging study. J Sleep Res. 2013;22(2):144-154.

96. Fischer S, Hallschmid M, Elsner AL, Born J. Sleep forms memory for finger skills. Proc Natl Acad Sci U S A. 2002;99(18):11987-11991.

97. Walker MP, Brakefield T, Morgan A, Hobson JA, Stickgold R. Practice with sleep makes perfect: sleep-dependent motor skill learning. Neuron. 2002;35(1):205-211.

98. Spencer RM, Gouw AM, Ivry RB. Age-related decline of sleepdependent consolidation. Learn Mem. 2007;14(7):480-484.

99. Kuriyama K, Stickgold R, Walker MP. Sleep-dependent learning and motor-skill complexity. Learn Mem. 2004;11(6):705-713.

100. Varga AW, Kang M, Ramesh PV, Klann E. Effects of acute sleep deprivation on motor and reversal learning in mice. Neurobiol Learn Mem. 2014;114:217-222.

101. Nagai H, de Vivo L, Bellesi M, Ghilardi MF, Tononi G, Cirelli C. Sleep consolidates motor learning of complex movement sequences in mice. Sleep. 2016;40(2).

102. Cohen DA, Pascual-Leone A, Press DZ, Robertson EM. Off-line learning of motor skill memory: a double dissociation of goal and movement. Proc Natl Acad Sci U S A. 2005;102(50):18237-18241.

103. Smith C. Sleep states and memory processes in humans: procedural versus declarative memory systems. Sleep Med Rev. 2001;5(6):491-506.

104. Smith CT, Nixon MR, Nader RS. Posttraining increases in REM sleep intensity implicate REM sleep in memory processing and provide a biological marker of learning potential. Learn Mem. 2004;11(6): 714-719.

105. Maquet P, Laureys S, Peigneux P, et al. Experience-dependent changes in cerebral activation during human REM sleep. Nat Neurosci. 2000;3(8):831-836.

106. Laureys S, Peigneux P, Phillips C, et al. Experience-dependent changes in cerebral functional connectivity during human rapid eye movement sleep. Neuroscience. 2001;105(3):521-525.

107. Nitsche MA, Jakoubkova M, Thirugnanasambandam N, et al. Contribution of the premotor cortex to consolidation of motor sequence learning in humans during sleep. J Neurophysiol. 2010;104(5):2603-2614.

108. Smith C. REM sleep and learning: some recent findings. In: Moffit A, Kramer M, editors. The Functions of Dreaming. Albany, NY: State University of New York Press; 1993.

109. Goerke M, Cohrs S, Rodenbeck A, Kunz D. Differential effect of an anticholinergic antidepressant on sleep-dependent memory consolidation. Sleep. 2014;37(5):977-985.

110. Rasch B, Pommer J, Diekelmann S, Born J. Pharmacological REM sleep suppression paradoxically improves rather than impairs skill memory. Nat Neurosci. 2009;12(4):396-397. 
111. Göder R, Seeck-Hirschner M, Stingele K, et al. Sleep and cognition at baseline and the effects of REM sleep diminution after 1 week of antidepressive treatment in patients with depression. J Sleep Res. 2011;20(4):544-551.

112. Fogel SM, Smith CT, Cote KA. Dissociable learning-dependent changes in REM and non-REM sleep in declarative and procedural memory systems. Behav Brain Res. 2007;180(1):48-61.

113. Nishida M, Walker MP. Daytime naps, motor memory consolidation and regionally specific sleep spindles. PLoS One. 2007;2(4):e341.

114. Yordanova J, Kirov R, Verleger R, Kolev V. Dynamic coupling between slow waves and sleep spindles during slow wave sleep in humans is modulated by functional pre-sleep activation. Sci Rep. 2017;7(1):14496.

115. Ackermann S, Rasch B. Differential effects of non-REM and REM sleep on memory consolidation? Curr Neurol Neurosci Rep. 2014;14(2):430.

116. Johnson LA, Blakely T, Hermes D, Hakimian S, Ramsey NF, Ojemann JG. Sleep spindles are locally modulated by training on a brain-computer interface. Proc Natl Acad Sci USA. 2012;109(45):18583-18588.

117. Lustenberger C, Boyle MR, Alagapan S, Mellin JM, Vaughn BV, Fröhlich F. Feedback-controlled transcranial alternating current stimulation reveals a functional role of sleep spindles in motor memory consolidation. Curr Biol. 2016;26(16):2127-2136.

118. Stickgold R, Walker MP. Sleep-dependent memory triage: evolving generalization through selective processing. Nat Neurosci. 2013;16(2):139-145.

119. Chatburn A, Lushington K, Kohler MJ. Complex associative memory processing and sleep: a systematic review and meta-analysis of behavioural evidence and underlying EEG mechanisms. Neurosci Biobehav Rev. 2014;47:646-655.

120. Saletin JM, Goldstein AN, Walker MP. The role of sleep in directed forgetting and remembering of human memories. Cereb Cortex. 2011;21(11):2534-2541.

121. Fischer S, Born J. Anticipated reward enhances offline learning during sleep. J Exp Psychol Learn Mem Cogn. 2009;35(6):1586-1593.

122. van Rijn E, Lucignoli C, Izura C, Blagrove MT. Sleep-dependent memory consolidation is related to perceived value of learned material. J Sleep Res. 2017;26(3):302-308.

123. van Dongen EV, Thielen JW, Takashima A, Barth M, Fernández G. Sleep supports selective retention of associative memories based on relevance for future utilization. PLoS One. 2012;7(8):e43426.

124. Rauchs G, Feyers D, Landeau B, et al. Sleep contributes to the strengthening of some memories over others, depending on hippocampal activity at learning. J Neurosci. 2011;31(7):2563-2568.

125. Payne JD, Schacter DL, Propper RE, et al. The role of sleep in false memory formation. Neurobiol Learn Mem. 2009;92(3):327-334.

126. Ellenbogen JM, Hu PT, Payne JD, Titone D, Walker MP. Human relational memory requires time and sleep. Proc Natl Acad Sci U SA. 2007;104(18):7723-7728.

127. Djonlagic I, Rosenfeld A, Shohamy D, Myers C, Gluck M, Stickgold R. Sleep enhances category learning. Learn Mem. 2009;16(12):751-755.

128. Barsky MM, Tucker MA, Stickgold R. REM sleep enhancement of probabilistic classification learning is sensitive to subsequent interference. Neurobiol Learn Mem. 2015;122:63-68.

129. Kirov R, Kolev V, Verleger R, Yordanova J. Labile sleep promotes awareness of abstract knowledge in a serial reaction time task. Front Psychol. 2015;6:1354.

130. Yordanova J, Kolev V, Bruns E, Kirov R, Verleger R. Sleep spindles in the right hemisphere support awareness of regularities and reflect pre-sleep activations. Sleep. 2017;40(11).

131. Kirov R. Editorial: brain oscillations and predictive coding in the context of different conscious states and sleep-wake cycle: implications for decision making and psychopathology. Front Psychol. 2016;7:1768. eCollection 2016.

132. Oudiette D, Paller KA. Upgrading the sleeping brain with targeted memory reactivation. Trends Cogn Sci. 2013;17(3):142-149.
133. Hars B, Hennevin E, Pasques P. Improvement of learning by cueing during postlearning paradoxical sleep. Behav Brain Res. 1985;18(3):241-250.

134. Smith C, Weeden K. Post training REMs coincident auditory stimulation enhances memory in humans. Psychiatr J Univ Ott. 1990;15(2):85-90.

135. Guerrien A, Dujardin K, Mandai O, Sockeel P, Leconte P. Enhancement of memory by auditory stimulation during postlearning REM sleep in humans. Physiol Behav. 1989;45(5):947-950.

136. Rudoy JD, Voss JL, Westerberg CE, Paller KA. Strengthening individual memories by reactivating them during sleep. Science. 2009;326(5956):1079.

137. Rasch B, Büchel C, Gais S, Born J. Odor cues during slowwave sleep prompt declarative memory consolidation. Science. 2007;315(5817):1426-1429.

138. Schreiner T, Rasch B. Boosting vocabulary learning by verbal cueing during sleep. Cereb Cortex. 2015;25(11):4169-4179.

139. Antony JW, Gobel EW, O'Hare JK, Reber PJ, Paller KA. Cued memory reactivation during sleep influences skill learning. Nat Neurosci. 2012;15(8):1114-1116.

140. Schönauer M, Geisler T, Gais S. Strengthening procedural memories by reactivation in sleep. J Cogn Neurosci. 2014;26(1):143-153.

141. Batterink LJ, Westerberg CE, Paller KA. Vocabulary learning benefits from REM after slow-wave sleep. Neurobiol Learn Mem. 2017;144:102-113.

142. Cousins JN, El-Deredy W, Parkes LM, Hennies N, Lewis PA. Cued reactivation of motor learning during sleep leads to overnight changes in functional brain activity and connectivity. PLoS Biol. 2016;14(5):e1002451.

143. Sterpenich V, Schmidt C, Albouy G, et al. Memory reactivation during rapid eye movement sleep promotes its generalization and integration in cortical stores. Sleep. 2014;37(6):1061-1075, 1075A-1075B.

144. Hu X, Antony JW, Creery JD, Vargas IM, Bodenhausen GV, Paller KA. Cognitive neuroscience. Unlearning implicit social biases during sleep. Science. 2015;348(6238):1013-1015.

145. Cedernaes J, Osorio RS, Varga AW, Kam K, Schiöth HB, Benedict C. Candidate mechanisms underlying the association between sleep-wake disruptions and Alzheimer's disease. Sleep Med Rev. 2017;31:102-111.

146. Djonlagic I, Saboisky J, Carusona A, Stickgold R, Malhotra A. Increased sleep fragmentation leads to impaired off-line consolidation of motor memories in humans. PLoS One. 2012;7(3):e34106.

147. Landry S, Anderson C, Andrewartha P, Sasse A, Conduit R. The impact of obstructive sleep apnea on motor skill acquisition and consolidation. J Clin Sleep Med. 2014;10(5):491-496.

148. Djonlagic I, Guo M, Matteis P, Carusona A, Stickgold R, Malhotra A. Untreated sleep-disordered breathing: links to aging-related decline in sleep-dependent memory consolidation. PLoS One. 2014;9(1):e85918.

149. Djonlagic I, Guo M, Matteis P, Carusona A, Stickgold R, Malhotra A. First night of CPAP: impact on memory consolidation, attention and subjective experience. Sleep Med. 2015;16(6):697-702.

150. Kloepfer C, Riemann D, Nofzinger EA, et al. Memory before and after sleep in patients with moderate obstructive sleep apnea. J Clin Sleep Med. 2009;5(6):540-548.

151. Csabi E, Varszegi-Schulz M, Janacsek K, Malecek N, Nemeth D. The consolidation of implicit sequence memory in obstructive sleep apnea. PLoS One. 2014;9(10):e109010.

152. Kheirandish-Gozal L, De Jong MR, Spruyt K, Chamuleau SA, Gozal D. Obstructive sleep apnoea is associated with impaired pictorial memory task acquisition and retention in children. Eur Respir J. 2010;36(1):164-169.

153. Maski K, Steinhart E, Holbrook H, Katz ES, Kapur K, Stickgold R. Impaired memory consolidation in children with obstructive sleep disordered breathing. PLoS One. 2017;12(11):e0186915.

154. Bucks RS, Olaithe M, Rosenzweig I, Morrell MJ. Reviewing the relationship between OSA and cognition: where do we go from here? Respirology. 2017;22(7):1253-1261. 
155. Neumann KF, Rojo L, Navarrete LP, Farías G, Reyes P, Maccioni RB. Insulin resistance and Alzheimer's disease: molecular links \& clinical implications. Curr Alzheimer Res. 2008;5(5):438-447.

156. Alzoubaidi M, Mokhlesi B. Obstructive sleep apnea during rapid eye movement sleep: clinical relevance and therapeutic implications. $\mathrm{Curr}$ Opin Pulm Med. 2016;22(6):545-554.

157. Beebe DW, Groesz L, Wells C, Nichols A, McGee K. The neuropsychological effects of obstructive sleep apnea: a meta-analysis of norm-referenced and case-controlled data. Sleep. 2003;26(3):298-307.

158. Stranks EK, Crowe SF. The cognitive effects of obstructive sleep apnea an updated meta-analysis. Arch Clin Neuropsychol. 2016;31(2):186-193.

159. Wallace A, Bucks RS. Memory and obstructive sleep apnea: a metaanalysis. Sleep. 2013;36(2):203-220.

160. Olaithe M, Bucks RS, Hillman DR, Eastwood PR. Cognitive deficits in obstructive sleep apnea: insights from a meta-review and comparison with deficits observed in COPD, insomnia, and sleep deprivation. Sleep Med Rev. 2018;38:39-49.

161. Kushida CA, Nichols DA, Holmes TH, et al. Effects of continuous positive airway pressure on neurocognitive function in obstructive sleep apnea patients: the Apnea Positive Pressure Long-term Efficacy Study (APPLES). Sleep. 2012;35(12):1593-1602.
162. Marcus CL, Moore RH, Rosen CL, et al; Childhood Adenotonsillectomy Trial (CHAT). A randomized trial of adenotonsillectomy for childhood sleep apnea. $N$ Engl J Med. 2013;368(25): 2366-2376.

163. Jackson ML, McEvoy RD, Banks S, Barnes M. Neurobehavioral impairment and CPAP treatment response in mild-moderate obstructive sleep apneas. J Clin Sleep Med. 2018;14(1):47-56.

164. Bliwise DL, Greenaway MC. Will APPLES hit a ceiling? Sleep. 2011;34(3):249-250.

165. Brouillette RT. Let's CHAT about adenotonsillectomy. $N$ Engl J Med. 2013;368(25):2428-2429.

166. Baril AA, Gagnon K, Brayet P, et al. Gray matter hypertrophy and thickening with obstructive sleep apnea in middle-aged and older adults. Am J Respir Crit Care Med. 2017;195(11):1509-1518.

167. Kumar R, Pham TT, Macey PM, Woo MA, Yan-Go FL, Harper RM. Abnormal myelin and axonal integrity in recently diagnosed patients with obstructive sleep apnea. Sleep. 2014;37(4):723-732.

168. Cha J, Zea-Hernandez JA, Sin S, et al. The effects of obstructive sleep apnea syndrome on the dentate gyrus and learning and memory in children. J Neurosci. 2017;37(16):4280-4288.
Nature and Science of Sleep

\section{Publish your work in this journal}

Nature and Science of Sleep is an international, peer-reviewed, open access journal covering all aspects of sleep science and sleep medicine, including the neurophysiology and functions of sleep, the genetics of sleep, sleep and society, biological rhythms, dreaming, sleep disorders and therapy, and strategies to optimize healthy sleep. The manuscript

\section{Dovepress}

management system is completely online and includes a very quick and fair peer-review system, which is all easy to use. Visit http://www. dovepress.com/testimonials.php to read real quotes from published authors. 